\title{
Scattering of a Tightly Focused Beam by an Optically Trapped Particle
}

James A. Lock

Cleveland State University, j.lock@csuohio.edu

Susan Y. Wrbanek

Kenneth E. Weiland

Follow this and additional works at: https://engagedscholarship.csuohio.edu/sciphysics_facpub

Part of the Physics Commons

How does access to this work benefit you? Let us know!

\section{Publisher's Statement}

This paper was published in Applied Optics and is made available as an electronic reprint with the permission of OSA. The paper can be found at the following URL on the OSA website: http://www.opticsinfobase.org/ao/abstract.cfm?URI=ao-45-15-3634. Systematic or multiple reproduction or distribution to multiple locations via electronic or other means is prohibited and is subject to penalties under law.

\section{Original Citation}

Lock, James A., Susan Y. Wrbanek, and Kenneth E. Weiland (retired). "Scattering of a Tightly Focused Beam by an Optically Trapped Particle." Applied Optics 45 (2006): 3634-3645.

\section{Repository Citation}

Lock, James A.; Wrbanek, Susan Y.; and Weiland, Kenneth E., "Scattering of a Tightly Focused Beam by an Optically Trapped Particle" (2006). Physics Faculty Publications. 90.

https://engagedscholarship.csuohio.edu/sciphysics_facpub/90

This Article is brought to you for free and open access by the Physics Department at EngagedScholarship@CSU. It has been accepted for inclusion in Physics Faculty Publications by an authorized administrator of EngagedScholarship@CSU. For more information, please contact library.es@csuohio.edu. 


\title{
Scattering of a tightly focused beam by an optically trapped particle
}

\author{
James A. Lock, Susan Y. Wrbanek, and Kenneth E. Weiland (retired)
}

\begin{abstract}
Near-forward scattering of an optically trapped 5- $\mu$ m-radius polystyrene latex sphere by the trapping beam was examined both theoretically and experimentally. Since the trapping beam is tightly focused, the beam fields superpose and interfere with the scattered fields in the forward hemisphere. The observed light intensity consists of a series of concentric bright and dark fringes centered about the forwardscattering direction. Both the number of fringes and their contrast depend on the position of the trapping beam focal waist with respect to the sphere. The fringes are caused by diffraction that is due to the truncation of the tail of the trapping beam as the beam is transmitted through the sphere. (C) 2006 Optical Society of America

OCIS codes: $140.7010,290.4020$.
\end{abstract}

\section{Introduction}

When a downward-propagating tightly focused laser beam is incident upon a small particle, the radiation force of the beam on the particle can sometimes be directed upward. If the upward radiation force is sufficiently strong, it can balance gravity and optically trap the particle. ${ }^{1}$ The radiation trapping force has been calculated by use of ray theory,,$^{2,3}$ Rayleigh scattering, ${ }^{1,4}$ Rayleigh-Gans scattering, ${ }^{5-7}$ and Mie theory. ${ }^{8-14}$ In each of these approaches one must model the details of the incident beam. The two beam types most commonly used in trapping calculations are (1) a freely propagating focused Gaussian beam in the medium surrounding the particle, paying no attention to the way in which the beam is produced, and (2) a Gaussian beam that overfills a high-numerical-aperture (NA) oil-immersion microscope objective lens and is transmitted from a microscope coverslip to a water-filled sample cell, thus acquiring spherical aberration. ${ }^{15-17}$ Although the Gaussian beam is highly idealized whereas the apertured, focused, and aberrated beam (hereafter called an AFA beam)

J. A. Lock is with the Physics Department, Cleveland State University, Cleveland, Ohio 44115. S. Y. Wrbanek and K. E. Weiland (retired) are with NASA, Glenn Research Center, Brookpark, Ohio, 44135.

Received 27 September 2005; accepted 23 November 2005; posted 12 December 2005 (Doc. ID 64995).

0003-6935/06/153634-12\$15.00/0

C 2006 Optical Society of America is more experimentally realistic, their predicted trapping properties are found to be surprisingly similar when the particle to be trapped is near the top of the sample cell where the spherical aberration of the AFA beam is small. ${ }^{14}$

In this paper we examine scattering of the trapping beam by a trapped particle and determine the conditions under which the near-forward-direction light-scattering signature of the freely propagating Gaussian beam and the AFA beam greatly differ in spite of the similarity of their trapping properties. A complication caused by the trapping beam's tight focus is that it has a wide angular extent in the far zone that substantially overlaps the scattered light.

Experimental measurements record the intensity of the beam-plus-scattered light, which necessitates calculating the beam's far-zone fields and adding them to the scattered fields. For weak focusing, the paraxial far-zone beam fields are well approximated by the Fourier transform of the beam fields in the plane containing the center of the particle. ${ }^{18-21}$ However, for strong focusing and wide angular spreading in the far zone, the paraxial approximation is inappropriate and an alternative approach is required.

The body of this paper is organized as follows. In Section 2 we describe a tightly focused on-axis beam and its far-zone asymptotic form in terms of the beam's partial-wave shape coefficients. We also give the specific form of the shape coefficients for a freely propagating focused Gaussian beam and an AFA beam. In Section 3 we compute the trapping properties of each of these beams for a 5- $\mu$ m-radius polystyrene latex (PSL) sphere in water. We find that 
both the maximum trapping efficiency and the trapping range are similar when each beam has the same focal waist radius and the sphere is near the top of the sample cell. We also find that for high laser power the center of the focal waist of each beam is predicted to lie near the center of the particle in the stable trapping position. Section 4 examines the calculated angular structure of the light scattered by the PSL sphere in the near-forward direction, the physical scattering mechanisms responsible for the structure, and the features of the structure that differ markedly for the tightly focused Gaussian beam and the AFA beam. We find that, when the center of the beam waist lies outside the sphere, scattering by each beam is similar. However, when the center of the beam waist lies deep inside the particle, the scattered light in the forward hemisphere for each of the two beams is quite different. In Section 5 we describe an experiment whose purpose was to measure the laser beam trapping length and to observe the near-forwardscattered intensity. Finally, in Section 6 we summarize our conclusions.

\section{On-Axis Beam Fields in the Far Zone}

\section{A. Beam Amplitudes}

Consider an on-axis focused electromagnetic beam in a medium of refractive index $n$. The beam propagates in the positive $z$ direction of a fixed coordinate system, and the beam axis coincides with the $z$ axis. The beam has free-space wavelength $\lambda$, wavenumber $k=2 \pi / \lambda$, and time dependence $\exp (-i \omega t)$. The center of the beam focal waist is located at $z=z_{0}$. In the beam's focal plane the peak electric-field strength is $E_{0}$ and the beam's electric field is polarized in the $x$ direction. The partial-wave decomposition of the beam electric field is ${ }^{13}$

$$
\begin{aligned}
\mathbf{E}_{\text {beam }}(r, \theta, \phi)= & -E_{0} \sum_{l=1}^{\infty} i^{l+1}(2 l+1) g_{l}\left[j_{l}(n k r) /(n k r)\right] \\
& \times \pi_{l}(\theta) \sin (\theta) \cos (\phi) \mathbf{u}_{r} \\
& +E_{0} \sum_{l=1}^{\infty}\left\{i^{l}(2 l+1) /[l(l+1)]\right\} \\
& \times\left[h_{l} j_{l}(n k r) \pi_{l}(\theta)-i g_{l} L_{l}(n k r) \tau_{l}(\theta)\right] \\
& \times \cos (\phi) \mathbf{u}_{\theta}-E_{0} \sum_{l=1}^{\infty}\left\{i^{l}(2 l+1) /\right. \\
& {[l(l+1)]\}\left[h_{l} j_{l}(n k r) \tau_{l}(\theta)\right.} \\
& \left.-i g_{l} L_{l}(n k r) \pi_{l}(\theta)\right] \sin (\phi) \mathbf{u}_{\phi},
\end{aligned}
$$

where $g_{l}$ and $h_{l}$ are the partial-wave shape coefficients of the beam, $j_{l}(n k r)$ are spherical Bessel functions,

$$
L_{l}(n k r) \equiv j_{l}(n k r) /(n k r)-j_{l}{ }^{\prime}(n k r),
$$

and the prime in Eq. (2) denotes the derivative of the spherical Bessel function with respect to its argument. The Mie theory angular functions are

$$
\begin{aligned}
\pi_{l}(\theta) & =P_{l}^{1}[\cos (\theta)] / \sin (\theta), \\
\tau_{l}(\theta) & =(\mathrm{d} / \mathrm{d} \theta) P_{l}^{1}[\cos (\theta)] .
\end{aligned}
$$

If the beam amplitude is circularly symmetric in its focal plane, the beam-shape coefficients satisfy $g_{l}=h_{l}$. To calculate the beam fields in the scattering far zone, the spherical Bessel functions in Eq. (1) are decomposed into incoming and outgoing spherical Hankel functions. ${ }^{22}$ When only the outgoing Hankel function for $\theta$ in the forward hemisphere is retained, the beam's electric field in the $r \rightarrow \infty$ far zone becomes

$$
\begin{aligned}
\mathbf{E}_{\text {beam }}(r, \theta, \phi)= & -i E_{0}[\exp (i n k r) /(n k r)] \\
& \times\left[-S_{2, \text { beam }}(\theta) \cos (\phi) \mathbf{u}_{\theta}\right. \\
& \left.+S_{1, \text { beam }}(\theta) \sin (\phi) \mathbf{u}_{\phi}\right]+O\left(1 / r^{2}\right),
\end{aligned}
$$

where the far-zone beam amplitudes are

$$
\begin{aligned}
S_{1, \text { beam }}(\theta)= & (-1 / 2) \sum_{l=1}^{\infty}\{(2 l+1) /[l(l+1)]\}\left[g_{l} \pi_{l}(\theta)\right. \\
& \left.+h_{l} \tau_{l}(\theta)\right], \\
S_{2, \text { beam }}(\theta)= & (-1 / 2) \sum_{l=1}^{\infty}\{(2 l+1) /[l(l+1)]\}\left[g_{l} \tau_{l}(\theta)\right. \\
& \left.+h_{l} \pi_{l}(\theta)\right] .
\end{aligned}
$$

A spherical particle of radius $a$ and refractive index $N$ has its center at the origin of coordinates and scatters the focused beam. In the far zone the scattered electric field is

$$
\begin{aligned}
\mathbf{E}_{\text {scatt }}(r, \theta, \phi)= & -i E_{0}[\exp (i n k r) /(n k r)] \\
& \times\left[-S_{2, \operatorname{scatt}}(\theta) \cos (\phi) \mathbf{u}_{\theta}\right. \\
& \left.+S_{1, \text { scatt }}(\theta) \sin (\phi) \mathbf{u}_{\phi}\right]+O\left(1 / r^{2}\right)
\end{aligned}
$$

where the scattering amplitudes are

$$
\begin{aligned}
S_{1, \text { scatt }}(\theta)= & \sum_{l=1}^{\infty}\{(2 l+1) /[l(l+1)]\}\left[a_{l} g_{l} \pi_{l}(\theta)\right. \\
& \left.+b_{l} h_{l} \tau_{l}(\theta)\right], \\
S_{2, \text { scatt }}(\theta)= & \sum_{l=1}^{\infty}\{(2 l+1) /[l(l+1)]\}\left[a_{l} g_{l} \tau_{l}(\theta)\right. \\
& \left.+b_{l} h_{l} \pi_{l}(\theta)\right],
\end{aligned}
$$

and $a_{l}$ and $b_{l}$ are the Mie theory partial-wave scattering amplitudes. ${ }^{23}$ If the beam is tightly focused in its focal plane, it has a wide angular spreading in the far zone. The beam fields and scattered fields then substantially overlap, and the total far-zone beamplus-scattered amplitudes are

$$
\begin{aligned}
& S_{1, \text { total }}(\theta)=S_{1, \text { beam }}(\theta)+S_{1, \text { scatt }}(\theta), \\
& S_{2, \text { total }}(\theta)=S_{2, \text { beam }}(\theta)+S_{2, \text { scatt }}(\theta) .
\end{aligned}
$$

The Debye series decomposition of the scattered 
light can be used to simplify Eqs. ( $8 a$ ) and ( $8 b)$. In the large-sphere or short-wavelength limit $2 \pi n a / \lambda \gg 1$, the various terms of the Debye series decomposition of the partial-wave scattering amplitudes,

$$
a_{l}, b_{l}=(1 / 2)\left[1-R_{l}^{\text {external }}-\sum_{p=1}^{\infty} T_{l}^{\text {in }}\left(R_{l}^{\text {internal }}\right)^{p-1} T_{l}^{\text {out }}\right],
$$

acquire the following physically intuitive meanings. ${ }^{24,25}$ An incoming partial wave $l$ is in part diffracted by the sphere $(1 / 2)$, it is in part externally reflected from the sphere surface $\left(-R_{l}^{\text {external }} / 2\right)$, and it is in part transmitted through the sphere $\left(-T_{l}^{\text {in }} T_{l}^{\text {out }} / 2\right)$ following $p-1$ internal reflections $\left[\left(R_{l}{ }^{\text {internal }}\right)^{p-1}\right]$. Explicit forms for the partial-wave transmission and reflection coefficients are given in Ref. 25. When Eqs. (5a), (5b), (7a), (7b), and (9) are substituted into Eqs. (8a) and (8b), the beam amplitude exactly cancels the diffracted part of the scattering amplitude. ${ }^{26}$ Thus when the beam and the scattered fields overlap, the total amplitudes of Eqs. (8a) and (8b) contain only external reflection and transmission through the sphere following all numbers of internal reflections.

\section{B. Shape Coefficients of Paraxial Beams}

If the electric-field half-width of the beam in its focal plane is $w$, the confinement parameter $s$ of the beam is defined as

$$
s \equiv 1 /(n k w) .
$$

If $s \ll 1$ the beam is loosely focused and remains paraxial as it propagates to the far zone. In this situation it is common practice to specify the beam by its presumed shape in the focal plane rather than by specifying it by its partial-wave shape coefficients $g_{l}$ and $h_{l}$. However, it is the shape coefficients that are required in Eqs. (7a) and (7b) for calculating scattering of the beam by the spherical particle. One way to determine the shape coefficients from the beam's focal waist profile is the localized model, ${ }^{27,28}$ which replaces $n k$ multiplied by the transverse coordinate $\rho^{\prime}$ of the beam fields in the $z=0$ plane with $l+1 / 2$. For use in Section 4, a weakly focused Gaussian beam with the center of its focal waist in the $z=0$ plane,

$$
\mathbf{E}_{\text {beam }}\left(\rho^{\prime}, 0\right)=E_{0} \exp \left(-\rho^{\prime 2} / w^{2}\right) \mathbf{u}_{x},
$$

diffracts to the far zone to give

$$
\begin{aligned}
\mathbf{E}_{\text {diffracted }}(r, \theta, \phi)= & \left(i E_{0} / 2 s^{2}\right)[\exp (i n k r) /(n k r)] \\
& \times \exp \left(-\theta^{2} / 4 s^{2}\right)\left[-\cos (\phi) \mathbf{u}_{\theta}\right. \\
& \left.+\sin (\phi) \mathbf{u}_{\phi}\right]
\end{aligned}
$$

and has the localized model shape coefficients

$$
g_{l}=h_{l}=\exp \left[-s^{2}(l+1 / 2)^{2}\right] \text {. }
$$

Similarly, the weakly focused paraxial beam in the $z=0$ plane,

$$
\mathbf{E}_{\text {beam }}\left(\rho^{\prime}, 0\right)=2 E_{0} J_{1}\left(n k \rho^{\prime} \alpha\right) /\left(n k \rho^{\prime} \alpha\right) \mathbf{u}_{x},
$$

diffracts to the far zone ${ }^{29}$ to give an apertured spherical wave with opening angle $\alpha$,

$$
\begin{aligned}
E_{\text {diffracted }}(r, \theta, \phi)= & {\left[-2 i E_{0} / \alpha^{2}\right][\exp (i n k r) /(n k r)] } \\
& \times\left[-\cos (\phi) \mathbf{u}_{\theta}+\sin (\phi) \mathbf{u}_{\phi}\right] \text { for } \theta<\alpha \\
= & 0 \text { for } \theta>\alpha
\end{aligned}
$$

and has the localized model shape coefficients

$$
g_{l}=h_{l}=2 J_{1}[(l+1 / 2) \alpha] /[(l+1 / 2) \alpha] .
$$

As will be seen in Subsection 2.C, if these beams are focused at $z=z_{0}$ rather than at $z=0$, the shape coefficients acquire a phase factor obtained when the beam is diffracted from $z=z_{0}$ to $z=0$.

The weakly focused beams generated by the shape coefficients of Eqs. (13) and (16) each contain many partial waves, but for different reasons. The shape coefficients of the weakly focused Gaussian beam damp out only after many partial waves since $s \ll 1$. One can expect that for a tightly focused beam with $s \approx 1$ and $z_{0} \approx 0$ only a few partial waves will contribute to Eq. (13). The shape coefficients of the apertured paraxial beam damp out only after many partial waves because of the sharp cutoff of the farzone field at $\theta \approx \alpha$. For a tightly focused beam for which $\alpha$ is large, one can expect that the beam will continue to require many partial waves in order to build up the sharpness of the far-zone angular cutoff. In the analysis of previous experiments in which a weakly focused beam was scattered by a spherical particle and the total light intensity in the nearforward direction was measured, ${ }^{18-21}$ the scattered fields were obtained with Eqs. (7a) and (7b) with the localized beam-shape coefficients while the far-zone beam fields were analytically modeled by diffraction or by some suitable extension of it. This cannot be done when the beam is tightly focused.

\section{Shape Coefficients of Tightly Focused Beams}

In general, the presumed shape of the electric field in the focal plane is not an exact solution of Maxwell's equations. However, the approximation to that shape given by the beam generated by the localized shape coefficients is an exact solution, thereby repairing the defect in the original beam description. This distinction is not important for a weakly focused beam since the presumed shape is already a close approximation to an exact solution of Maxwell's equations. However, for strongly focused beams for which $s \approx 1$, the presumed shape increasingly differs from an exact solution, and the paraxial diffractive modeling of the evolution of the beam to the far zone becomes increasingly invalid as well. One alternative is to start by 


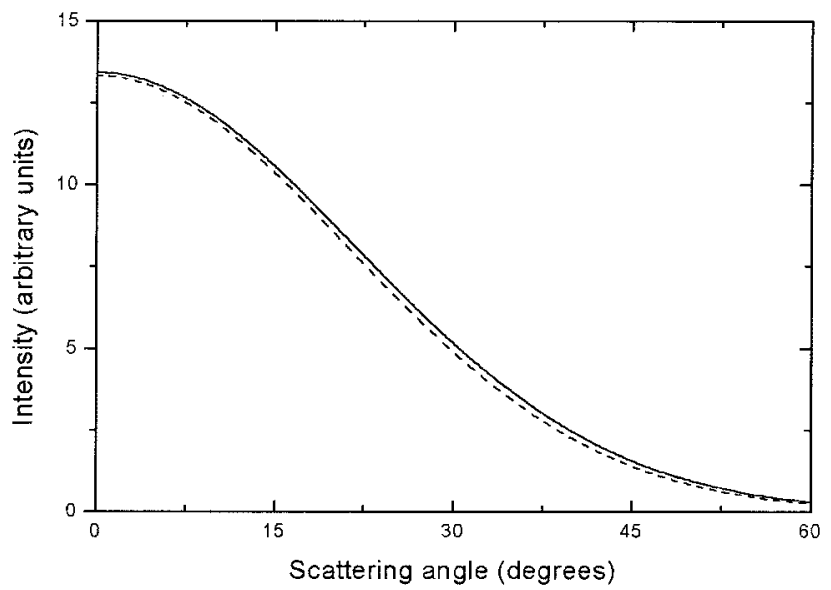

Fig. 1. Far-zone beam intensity (solid curve) as a function of the scattering angle in water $\theta$ for the freely propagating Gaussian beam generated from the shape coefficients of Eqs. (17) and (18) with $n=1.33, \lambda=0.532 \mu \mathrm{m}, w=0.172 \mu \mathrm{m}, w_{a}=0.205 \mu \mathrm{m}, z_{0}=$ $-1.60 \mu \mathrm{m}$, and $l_{\max }=97$. The dashed curve is the far-zone paraxial approximation of Eq. (12) with the intended width $w$.

specifying the beam-shape coefficients. In this section we apply the shape coefficients that were appropriate to a weakly focused beam without change to a strongly focused beam. The resulting beam is an exact solution of Maxwell's equations. However, the behavior of the tightly focused beam generated from the shape coefficients contains various distortions with respect to the paraxial behavior of the analogous weakly focused beam. Reference 13 examined distortions in the beam's focal plane. It was found that for both a strongly focused Gaussian beam and an AFA beam, the actual focal plane beam half-width $w_{a}$ was somewhat larger than the intended width $w$. As a result, when modeling a beam with a desired actual width, the intended width used as in input parameter in $g_{l}$ and $h_{l}$ is chosen to be somewhat smaller.

Here we examine the far-zone distortions of a tightly focused Gaussian beam and an AFA beam. If the Gaussian beam has the intended half-width $w$ and focuses at $z=z_{0}$, the localized beam-shape coefficients are $^{14}$

$$
g_{l}=h_{l}=D \exp \left(-i n k z_{0}\right) \exp \left[-D s^{2}(l+1 / 2)^{2}\right]
$$

where

$$
D=\left(1-2 i s z_{0} / w\right)^{-1} .
$$

The Gaussian beam examined here had $n=1.33$, $\lambda=0.532 \mu \mathrm{m}$, and an intended focal plane half-width of $w=0.172 \mu \mathrm{m}$, corresponding to an actual focal plane half-width of $w_{a}=0.205 \mu \mathrm{m}$, which is the focal waist width of a NA $=1.25$ oil-immersion microscope objective lens. The far-zone beam was calculated with Eqs. (5a), (5b), (17), and (18) for a number of values of $z_{0}$ near zero. Figure 1 shows the beam profile for $z_{0}=-1.60 \mu \mathrm{m}$ (a typical value) truncating the partial-wave series at $l_{\max }=97$. The reconstructed far-zone beam for $0^{\circ} \leq \theta \leq 60^{\circ}$ is Gaussian in shape and has an angular width corresponding to the intended width. Thus the tightly focused Gaussian beam generated by the shape coefficients of Eqs. (17) and (18) behaves in the far zone as if its width is $w$ and in the focal plane as if its width is $w_{a}$.

The AFA beam models a Gaussian beam of initial width $W$ that is incident upon and overfills a high-NA microscope objective lens of focal length $F$ and aperture radius $A$. Before focusing, it crosses an interface from a glass microscope coverslip with refractive index $n_{1}$ into water with refractive index $n_{2}$, thus acquiring spherical aberration. The coordinate of the center of the beam focal waist in the absence of the interface is $z_{0}$, and the coordinate of the interface is $d$ with $d<z_{0}$. The beam fields were modeled in Refs. $15-17$ by use of an angular spectrum of plane waves. The localized beam-shape coefficients derived from them are $^{14}$

$$
\begin{aligned}
g_{l}= & \left(-i n_{1} k F / 2\right) \int_{0}^{\alpha} \sin \left(\theta_{1}\right) \mathrm{d} \theta_{1}\left[\cos \left(\theta_{1}\right)\right]^{1 / 2} \\
& \times \exp \left\{-i\left[n_{2} k d \cos \left(\theta_{2}\right)+n_{1} k\left(z_{0}-d\right) \cos \left(\theta_{1}\right)\right]\right\} \\
& \times \exp \left[-(A / W)^{2} \tan ^{2}\left(\theta_{1}\right) / \tan ^{2}(\alpha)\right] \\
& \times\left\{\left[t_{\mathrm{TE}}+t_{\mathrm{TM}} \cos \left(\theta_{2}\right)\right] J_{0}\left[(l+1 / 2) \sin \left(\theta_{2}\right)\right]\right. \\
& \left.+\left[t_{\mathrm{TE}}-t_{\mathrm{TM}} \cos \left(\theta_{2}\right)\right] J_{2}\left[(l+1 / 2) \sin \left(\theta_{2}\right)\right]\right\}, \quad(19 a) \\
h_{l}= & \left(-i n_{1} k F / 2\right) \int_{0}^{\alpha} \sin \left(\theta_{1}\right) \mathrm{d} \theta_{1}\left[\cos \left(\theta_{1}\right)\right]^{1 / 2} \\
& \times \exp \left\{-i\left[n_{2} k d \cos \left(\theta_{2}\right)+n_{1} k\left(z_{0}-d\right) \cos \left(\theta_{1}\right)\right]\right\} \\
& \times \exp \left[-(A / W)^{2} \tan ^{2}\left(\theta_{1}\right) / \tan ^{2}(\alpha)\right] \\
& \times\left\{\left[t_{\mathrm{TM}}+t_{\mathrm{TE}} \cos \left(\theta_{2}\right)\right] J_{0}\left[(l+1 / 2) \sin \left(\theta_{2}\right)\right]\right. \\
& \left.+\left[t_{\mathrm{TM}}-t_{\mathrm{TE}} \cos \left(\theta_{2}\right)\right] J_{2}\left[(l+1 / 2) \sin \left(\theta_{2}\right)\right]\right\}, \quad(19 \mathrm{~b})
\end{aligned}
$$

where the NA of the lens is

$$
\mathrm{NA}=n_{1} \sin (\alpha)
$$

the angles $\theta_{1}$ and $\theta_{2}$ of a component plane wave in the angular spectrum in glass and water are related by Snell's law,

$$
n_{1} \sin \left(\theta_{1}\right)=n_{2} \sin \left(\theta_{2}\right),
$$

and the Fresnel coefficients of a component plane wave for transmission through the glass-water interface are

$$
\begin{aligned}
t_{\mathrm{TE}} & =2 \cos \left(\theta_{1}\right) /\left[\cos \left(\theta_{1}\right)+\left(n_{2} / n_{1}\right) \cos \left(\theta_{2}\right)\right], \\
t_{\mathrm{TM}} & =2 \cos \left(\theta_{1}\right) /\left[\left(n_{2} / n_{1}\right) \cos \left(\theta_{1}\right)+\cos \left(\theta_{2}\right)\right] .
\end{aligned}
$$

The apertured beam considered here had $W / A$ $=5.0$, which approximates a plane wave incident upon the focusing lens, $n_{1}=1.50, n_{2}=1.33$, $\lambda=0.532 \mu \mathrm{m}$, and $\mathrm{NA}=1.25$, corresponding to trun- 


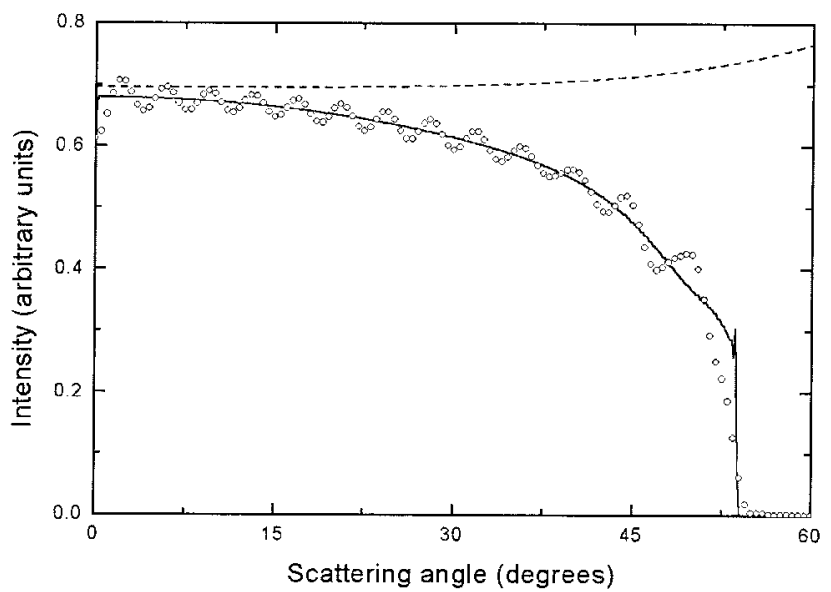

Fig. 2. Far-zone beam intensity (solid curve) as a function of the scattering angle in water $\theta_{2}$ for the AFA beam generated from the shape coefficients of Eqs. (19)-(22) with $W / A=5.0, n=1.50$, $n_{2}=1.33, \lambda=0.532 \mu \mathrm{m}, \mathrm{NA}=1.25, z_{0}=0.13 \mu \mathrm{m}, d=-4.95 \mu \mathrm{m}$, and $l_{\max }=1200$. The beam cuts off at $\theta_{2}=53.8^{\circ}$. The dashed curve is the far-zone paraxial approximation of Eq. (23), and the open circles are the reconstructed intensity when $l_{\max }=97$.

cating the converging beam in the glass at an angle of $\alpha=56.4^{\circ}$. The focal waist half-width in the absence of the interface is $0.202 \mu \mathrm{m}$. The reconstructed AFA beam in the far-zone was computed with Eqs. (5a), (5b), (19a), and (19b) with $l_{\max }=1200$. A large number of partial waves was required for accurately reconstructing the sharp cutoff of the far-zone beam. Figure 2 shows the intensity of the reconstructed beam for $z_{0}=0.13 \mu \mathrm{m}$ (a typical value). It also shows the paraxial approximation

$$
\begin{aligned}
\left|S_{1, \text { diffracted }}(\theta)\right|^{2}= & \left(n_{2} / n_{1}\right)^{4} \\
& \times\left[t_{\mathrm{TE}}+t_{\mathrm{TM}} \cos \left(\theta_{2}\right)\right]^{2} /\left[4 \cos \left(\theta_{1}\right)\right],
\end{aligned}
$$

and the intensity resulting when the beam reconstruction is truncated at $l_{\max }=97$. For $\alpha=56.4^{\circ}$ in glass, Snell's law predicts that the cutoff angle in water should be $\theta_{2}=69.9^{\circ}$. Figure 2 , however, shows that the cutoff of the reconstructed beam is $\theta_{2}=53.8^{\circ}$, corresponding to

$$
\theta_{2}=\left(n_{1} / n_{2}\right) \sin (\alpha)
$$

rather than Snell's law, as in Eq. (21). As a check of this result, a number of other apertured beams with $30^{\circ} \leq \alpha \leq 60^{\circ}$ were reconstructed from the shape coefficients of Eqs. (19a) and (19b). The cutoff was also found to be given by Eq. (24) rather than by Eq. (21). Figure 2 also shows that the reconstructed beam slowly decreases as a function of angle rather than remaining approximately constant since the beam incident upon the focusing lens approximates a plane wave. For the beam parameters considered here, the beam's calculated falloff is roughly Gaussian with $W / A \approx 1.5$. Thus if a Gaussian beam of specified half-width $W$ were incident upon the
high-NA lens, the far-zone reconstructed beam would be that of an incident Gaussian beam with a somewhat smaller half-width. This distortion is not expected to be important for trapping calculations since the trapping properties of the beam were found in Ref. 14 to be relatively insensitive to $W / A$.

\section{Trapping of a Spherical Particle by a Tightly Focused Beam}

An on-axis tightly focused Gaussian beam with $n=1.33, \lambda=0.532 \mu \mathrm{m}, w=0.172 \mu \mathrm{m}$, and $w_{a}=$ $0.205 \mu \mathrm{m}$, whose focal waist center is at $z_{0}$ in the absence of the particle, is incident from above upon a PSL sphere of radius $a=4.987 \mu \mathrm{m}$ and refractive index $N=1.59$ whose center is at the origin of coordinates. The computed radiation trapping force ${ }^{14}$ is directed upward for $-8.4 \mu \mathrm{m} \leq z_{0} \leq-1.5 \mu \mathrm{m}$. The beam focal point on the surface lies inside the sphere for $z_{0} \geq-5 \mu \mathrm{m}$, and it lies outside the sphere for $z_{0} \leq-5 \mu \mathrm{m}$. The maximum trapping efficiency of Eq. (8) of Ref. 14 was calculated to be $Q_{\max }=$ -0.0313 at $z_{0}=-4.8 \mu \mathrm{m}$. If the incident-beam power is larger than $\sim 60 \mathrm{~mW}$ with the relative density of PSL with respect to water of $1.05 \mathrm{~g} / \mathrm{cm}^{3}$, gravity can effectively be neglected with respect to the radiation trapping force and the stable trapping position should be near $z_{0}=-1.5 \mu \mathrm{m}$. As the beam power is decreased to $2 \mathrm{~mW}$, the effects of gravity become more important and the stable trapping position should move toward $z_{0}=-4.8 \mu \mathrm{m}$. If the beam is momentarily blocked when the stable trapping position is $z_{0} \approx-1.5 \mu \mathrm{m}$, the PSL sphere will start to fall through the water. If the beam is unblocked a short time later, it should be able to pull the sphere back up to the original trapping position as long as the radiation trapping force is still directed upward, i.e., if the sphere has fallen less than $\sim 6.9 \mu \mathrm{m}$.

For comparison, an AFA beam with $n_{1}=1.50$, $n_{2}=1.33, W / A=1.5, \lambda=0.532 \mu \mathrm{m}$, and NA = 1.25 is incident from above upon the same PSL sphere. The center of the sphere is still at the origin of coordinates, the center of the beam focal waist in the absence of both the glass-water interface and the particle is $z_{0}$, and the interface is located at $d=z_{0}$ $-5.08 \mu \mathrm{m}$. This corresponds to the stable trapping position for large laser power being at the top of the sample cell so that the spherical aberration produced by the glass-water interface is relatively small. The computed radiation trapping force is calculated to be directed upward for $-6.30 \mu \mathrm{m} \leq z_{0} \leq 0.08 \mu \mathrm{m}$. If $z_{0 p}$ and $z_{0 e}$ are the focal points of the paraxial and edge rays, respectively, of the aberrated beam in the water in the absence of the particle, this trapping range corresponds to $-6.88 \mu \mathrm{m} \leq z_{0 p} \leq-0.50 \mu \mathrm{m}$ and $-7.57 \mu \mathrm{m} \leq z_{0 e} \leq-1.19 \mu \mathrm{m}$, and as a result the beam's spherical aberration caustic thus lies inside the particle for $\sim 60 \%$ of the trapping range and is outside the particle for the other $40 \%$. The maximum trapping efficiency was calculated to be $Q_{\max }$ $=-0.0267$ at $z_{0}=-2.34 \mu \mathrm{m}$. The stable trapping position for large laser power is $z_{0} \approx 0.08 \mu \mathrm{m}$. The 


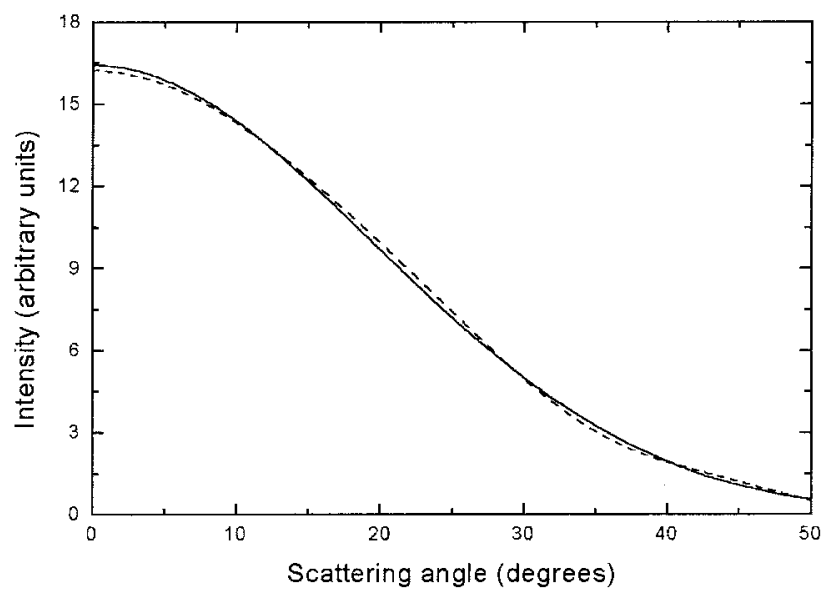

(a)

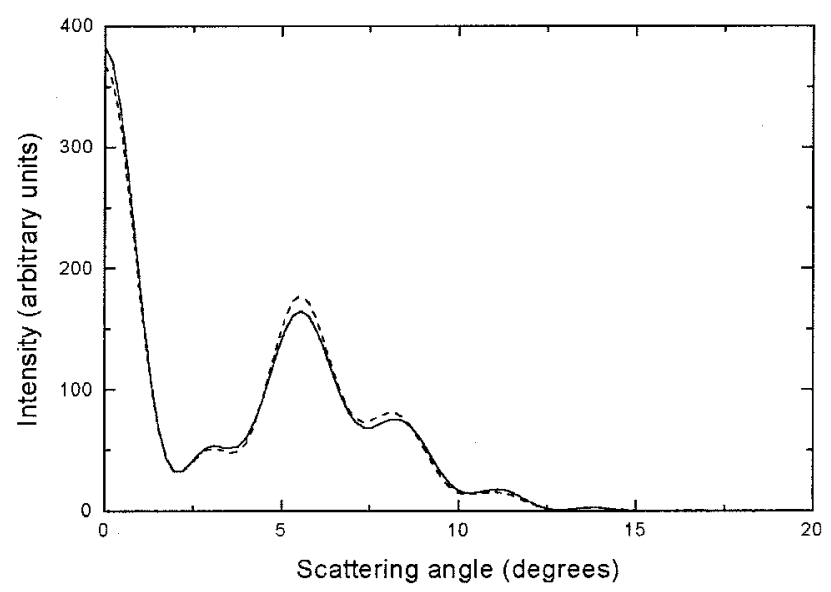

(c)

trapping range of $6.38 \mu \mathrm{m}$ differs from that of the Gaussian beam by only $7 \%$, and the maximum trapping efficiency differs by $15 \%$. As was found to be the case in Ref. 14, although the tightly focused Gaussian beam is highly idealized from an experimental point of view while the AFA beam is much more realistic, both beams have similar trapping properties for an $a=4.987 \mu \mathrm{m}$ PSL sphere in water near the top of the sample cell. If the sphere were trapped deeper in the sample cell, the predicted trapping properties of the two beam models would differ since the AFA beam takes into account the increasing spherical aberration of the beam at the glass-water interface whereas the Gaussian beam model does not. The trapping properties of the AFA beam were computed for $-40 \mu \mathrm{m} \leq d \leq-5 \mu \mathrm{m}$. It was found that although the maximum trapping efficiency decreased because of the increased spherical aberration as the sphere was farther from the glass-water interface, the calculated trapping length varied by less than $6 \%$.

\section{Scattering of a Tightly Focused Beam}

Since the tightly focused beams considered in Subsection 2.C have a far-zone angular half-width of $40^{\circ}-50^{\circ}$, the beam strongly overlaps the scattered light for much of the forward hemisphere. Thus the

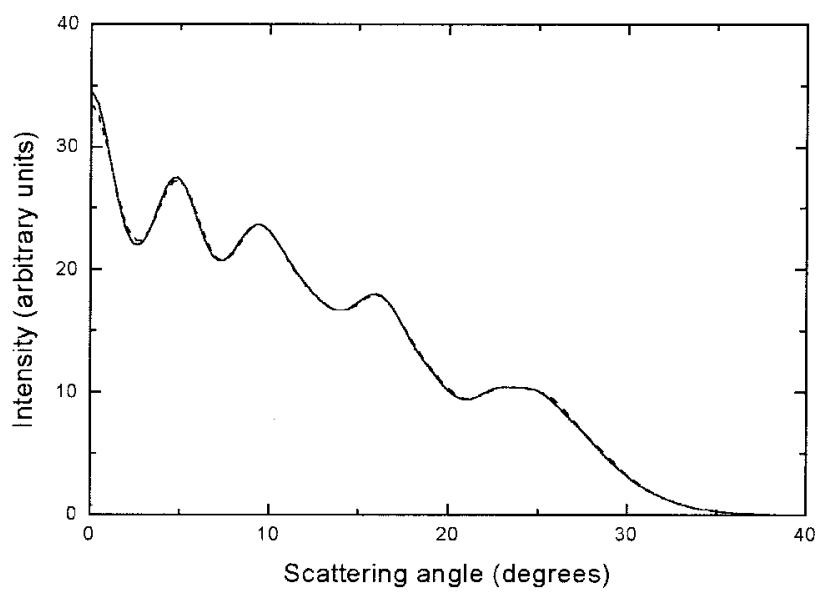

(b)

Fig. 3. Far-zone beam-plus-scattered intensity (solid curve) as a function of the scattering angle in water $\theta$ for the freely propagating Gaussian beam of Fig. 1 incident upon a PSL sphere with $a=4.987 \mu \mathrm{m}$ and $N=1.59$ for (a) $z_{0}=-1.60 \mu \mathrm{m}$, (b) $z_{0}=-4.32 \mu \mathrm{m}$, and (c) $z_{0}=-7.72 \mu \mathrm{m}$. The dashed curve is the intensity for the transmitted term of the Debye series expansion of the scattered light.

experimentally measured intensity corresponds to the beam-plus-scattering amplitudes of Eqs. 8(a) and $8(\mathrm{~b})$. We have already seen that, since the beam is tightly focused, its far-zone fields can differ greatly from those of the diffractive paraxial approximation. As a result, we obtained the incident beam used in Eqs. (8a) and (8b) by summing over partial waves in Eqs. (5a) and (5b) until convergence was obtained rather than by using an analytic expression for the diffracted fields as was done when the beam was loosely focused. ${ }^{20,21}$

The total intensity as a function of scattering angle $\theta$ in water was computed for the $a=4.987 \mu \mathrm{m}$ PSL sphere trapped in the tightly focused Gaussian beam of Fig. 1 for a number of values of $z_{0}$ in the trapping region. Representative results are shown in Figs. 3(a)-3(c). Since the half-width of the beam for small $z_{0}$ is much less than the particle's diameter, scattering for $\theta \leq 40^{\circ}$ should be dominated by transmission with no internal reflections. To verify this, Figs. 3(a)3(c) also show the intensity of the transmitted Debye series component of the scattered light. As expected, the transmitted intensity and the beam-plusscattered intensity are almost identical, providing a self-consistency check on the calculation.

Figure 3(a) shows the scattered intensity for 
$z_{0}=-1.60 \mu \mathrm{m}$. Assuming that the paraxial expression for the beam width as a function of position in the absence of the particle,

$$
w(z)=w\left[1+4\left(z-z_{0}\right)^{2} /\left(n k w^{2}\right)^{2}\right]^{1 / 2},
$$

remains at least qualitatively valid for a tightly focused beam, the half-width of the rapidly expanding Gaussian beam in the exit plane of the sphere for $z_{0}=-1.60 \mu \mathrm{m}$ is still smaller than the sphere radius. Thus the entire beam passes through the sphere, resulting in the lack of diffractive structure in the beam-plus-scattered light. Figure 3(b) shows the scattered intensity for $z_{0}=-4.32 \mu \mathrm{m}$. This beam focuses just inside the particle. Almost two radii later, when it exits, the width of the rapidly expanding beam is somewhat larger than the particle radius. Thus a part of the beam tail is effectively cut off by the particle, producing the diffractive structure superimposed on the Gaussian shape. These results may also be thought of from a complementary point of view. When $z_{0} \gg w / 2 s$, the Gaussian beam-shape coefficients of Eqs. (17) and (18) become

$$
\begin{aligned}
g_{l}=h_{l} \approx & \left(i w / 2 s z_{0}\right) \exp \left(-i n k z_{0}\right) \\
& \times \exp \left[-i s w(l+1 / 2)^{2} / 2 z_{0}\right] \\
& \times \exp \left[-w^{2}(l+1 / 2)^{2} / 4 z_{0}{ }^{2}\right] .
\end{aligned}
$$

When the particle radius is $a=4.987 \mu \mathrm{m}$, the largest partial wave included in the Mie theory scattering amplitudes of Eqs. (7a) and (7b) is

$$
l_{\max }=1+(2 \pi n a / \lambda)+4.3(2 \pi n a / \lambda)^{1 / 3}=97 .
$$

For $z_{0}=-1.6 \mu \mathrm{m}$, the Gaussian factor in Eq. (26) evaluated at $l_{\max }=97$ is $10^{-12}$, while for $z_{0}=-4.32$ $\mu \mathrm{m}$ the Gaussian factor has fallen to only 0.023 . This indicates that when the Mie sum for the scattered wave in Eqs. (7a) and (7b) is truncated at $l_{\max }$ the beam is completely reconstructed for the first value of $z_{0}$, thus producing a smooth scattering pattern, while important partial waves contributing to the beam shape are missing for the second value of $z_{0}$, thus producing the diffractive angular structure.

To estimate the scattering angle at which transmission effectively ceases in Figs. 3(a) and 3(b), the trajectory of the family of geometrical rays approximating the beam and crossing the $z$ axis inside the sphere was calculated. The scattering angle of the incident ray making an angle $42.4^{\circ}$ with the $z$ axis (i.e., the $1 / e^{2}$ intensity ray of the far-zone beam) for $z_{0}=-1.60 \mu \mathrm{m}$ was $\theta=38.3^{\circ}$, and for $z_{0}$ $=-4.32 \mu \mathrm{m}$ the scattering angle was $\theta=29.4^{\circ}$. These angles agree well with the $1 / e^{2}$ intensity points of the transmitted light in Figs. 3(a) and 3(b) in spite of the fact that, for $2 \pi n a / \lambda=78$, ray theory is only qualitatively accurate at best. In Refs. 20 and 21 the intensity oscillations in the beam-plus-scattered light for scattering by a weakly focused beam were due to an interference effect arising from the fact that the



Fig. 4. Far-zone beam-plus-scattered intensity (solid curve) for $\theta$ $=0^{\circ}$ as a function of the beam focal point location $z_{0}$ for the freely propagating Gaussian beam of Fig. 1 incident upon the PSL sphere of Fig. 3. The dashed curve is the $\theta=0^{\circ}$ intensity for the transmitted term of the Debye series expansion of the scattered light.

beam fields originate at $z=z_{0}$ while the scattered fields originate at $z=0$, and the surfaces of constant phase of the beam fields and the scattered fields reach the detector with different radii of curvature. This interference effect does not contribute to the intensity oscillations for $z_{0}=-4.32 \mu \mathrm{m}$ since the predicted angular sizes of the fringes because of different radii of curvature are an order of magnitude larger than those appearing in Fig. 3(b). Last, Fig. 3(c) shows the scattered light for $z_{0}=-7.72 \mu \mathrm{m}$. The source is now outside the particle, and when the beam is modeled by ray theory the transmitted light produces a rainbow-enhanced forward glory ${ }^{30,31}$ as long as $z_{0}$ $\geq-15.3 \mu \mathrm{m}$. The scattering angle of the rainbow accompanying the forward glory was computed with ray theory ${ }^{32}$ and occurs at $\theta=9.7^{\circ}$ for $z_{0}=-7.72 \mu \mathrm{m}$, agreeing well with the angular cutoff of the scattered intensity in Fig. 3(c).

Figure 4 shows the intensity for $\theta=0^{\circ}$ as a function of $z_{0}$ throughout the trapping region. When the beam focuses inside the particle and the entire beam fits within the particle's aperture $\left(z_{0} \geq-4 \mu \mathrm{m}\right)$, the forward intensity is rather featureless. However, when the beam focuses outside the particle $\left(z_{0} \leq-5 \mu \mathrm{m}\right)$, the forward intensity oscillates between bright and dark with intensity maxima occurring at $z_{0}=-5.85,-7.45$, and $-11.28 \mu \mathrm{m}$, and intensity minima at $z_{0}=-5.33,-6.41$, and $-8.31 \mu \mathrm{m}$. This general behavior is reminiscent of glory scattering. At each of these maxima and minima, the optical pathlength difference $\Delta L$ of the glory ray and the central ray was computed in ray theory. ${ }^{32} \mathrm{As} z_{0}$ recedes from the sphere surface, the difference between $\Delta L$ evaluated at adjacent maxima and minima for a glory should be $0.5 \lambda$, whereas in Fig. 4 it is $0.71 \lambda$, $0.58 \lambda, 0.75 \lambda, 0.43 \lambda$, and $0.76 \lambda$. This rough agreement suggests that the oscillations in the $\theta=0^{\circ}$ intensity as functions of the distance from the effective point source to the sphere surface are qualita- 


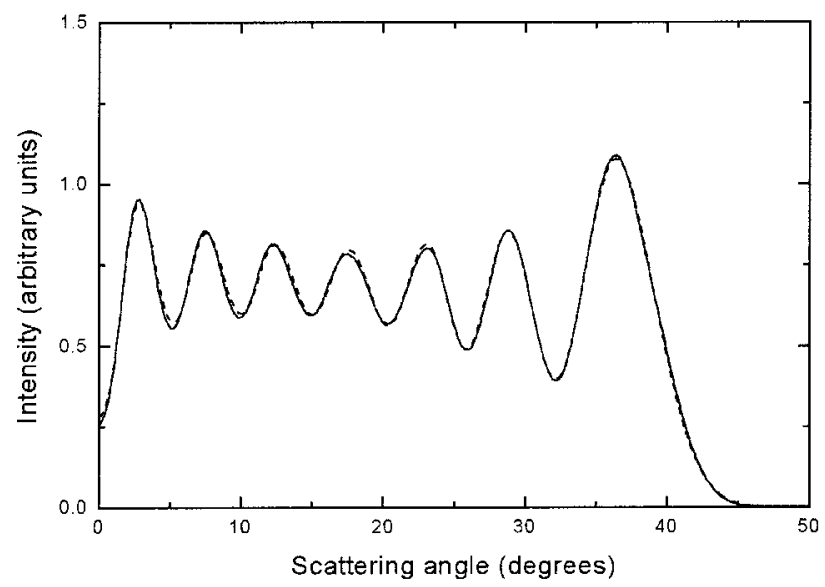

(a)

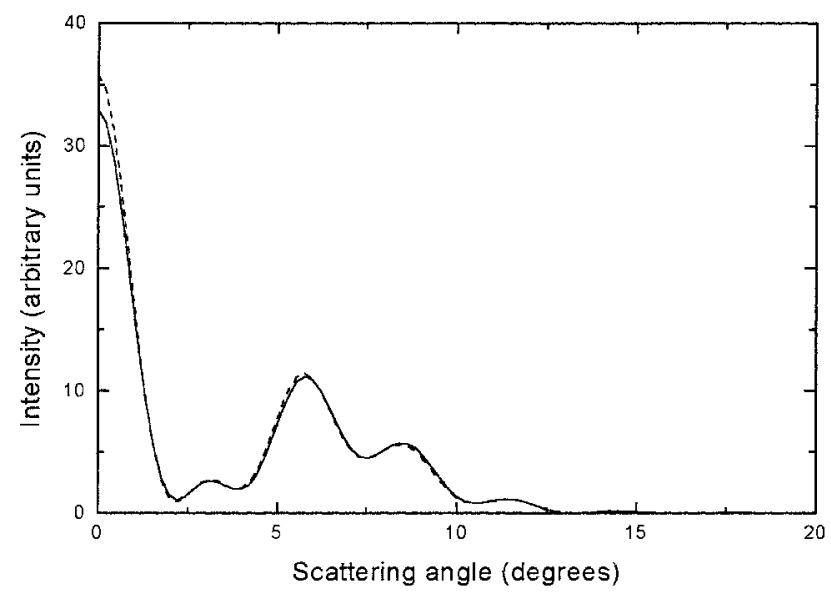

(c)

tively described by the interference between the glory ray and the central ray.

Figures 5(a)-5(c) show representative results for scattering by the AFA beam of Fig. 2. Figure 5(a) displays the scattered intensity for $z_{0}=0.08 \mu \mathrm{m}$. The scattered light in this figure consists of a number of concentric rings and cuts off at $\sim 42^{\circ}$. Since $l_{\max }$ $=97$ for scattering by the $a=4.987 \mu \mathrm{m}$ PSL sphere while 1200 partial waves are required for accurately reconstructing the sharp cutoff of the beam in the far zone, the sphere's effective aperture cuts off the tail of the beam in the $z=0$ plane, and the intensity ripples in the far-zone truncated beam in Fig. 2 are mirrored in the far-zone scattering pattern of Fig. 5(a). Figure 5(b) shows the scattered intensity for $z_{0}=-2.52 \mu \mathrm{m}$. There are now fewer concentric intensity rings and the cutoff of the scattered light occurs at a smaller angle $\theta$. These same trends were found in the reconstructed far-zone beam in the absence of the particle when the beam was truncated at 97 partial waves. Figure 5(c) shows the scattered intensity for $z_{0}=-6.30 \mu \mathrm{m}$ for which the spherical aberration caustic now lies outside the particle. As was the case for the focused Gaussian beam, this situation may be qualitatively described by an exterior point source that produces a rainbow-enhanced

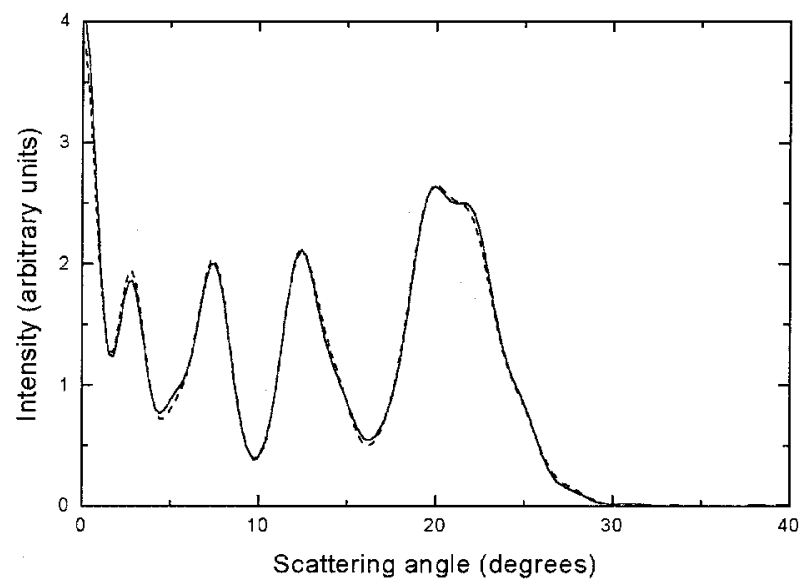

(b)

Fig. 5. Far-zone beam-plus-scattered intensity (solid curve) as a function of the scattering angle in water $\theta_{2}$ for the AFA beam of Fig. 2 (except with $W / A=1.5$ and new values of $d$ based on the value of $z_{0}$ ) incident upon the PSL sphere of Fig. 3 for (a) $z_{0}=0.08$ $\mu \mathrm{m}$, (b) $z_{0}=-2.52 \mu \mathrm{m}$, and (c) $z_{0}=-6.30 \mu \mathrm{m}$. The dashed curve is the intensity for the transmitted term of the Debye series expansion of the scattered light.

forward glory of the transmitted light. The details of the forward glory for the AFA beam differ somewhat from those of the Gaussian beam because of the blurring of the effective point source by the spherical aberration of the beam. Figure 6 shows the $\theta=0^{\circ}$ intensity as a function of $z_{0}$ throughout the trapping region. As opposed to the focused Gaussian beam case of Fig. 4, here the forward intensity oscillates between bright and dark for beam focusing both inside and outside the particle, giving a smooth transition between the diffraction behavior when the beam focal point lies inside the sphere and the sphere cuts off the tail of the trapping beam, and the rainbow-enhanced glory behavior when the focal point lies outside.

\section{Experiment}

We made a sample cell by creating a narrow slit approximately $3.17 \mathrm{~mm}$ wide between two pieces of laboratory film that were melted slightly to form walls between a clean microscope slide and a coverslip. The distilled water solution containing $a$ $=4.987 \pm 0.030 \mu \mathrm{m}$ PSL spheres at a volume fraction of $\sim 10^{-3}$ was drawn into the chamber by means of capillary action, and then the open ends of the 


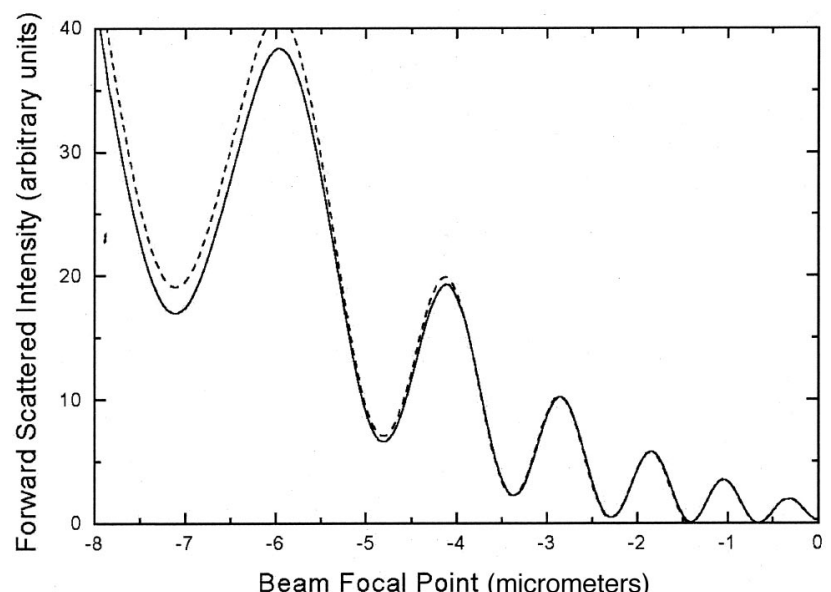

Fig. 6. Far-zone beam-plus-scattered intensity (solid curve) for $\theta_{2}$ $=0^{\circ}$ as a function of the beam focal point location $z_{0}$ for the AFA beam of Fig. 5 incident upon the PSL sphere of Fig. 3. The dashed curve is the $\theta_{2}=0^{\circ}$ intensity for the transmitted term of the Debye series expansion of the scattered light.

chamber were sealed with fingernail polish to prevent evaporation.

We measured the height of the water layer on the slide by using a micrometer and by viewing fiducial marks on the top of the slide and bottom of the coverslip through a microscope fitted with a $40 \times$ objective lens. The water layer height was found to be between $123 \pm 3$ and $140 \pm 4 \mu \mathrm{m}$ for different sample cells. The trapping beam was a downwardpropagating collimated Gaussian beam of a $\mathrm{Nd}$ : Vanadate laser with $\lambda=0.532 \mu \mathrm{m}$, operating at a power of $\sim 25 \mathrm{~mW}$, that slightly overfilled a NA $=1.25100 \times$ oil-immersion microscope objective lens and was focused within the sample cell, which was in optical contact with the objective lens by means of an index-matching oil.

The terminal velocity of a PSL sphere falling in the sample cell was measured as follows. A single sphere was optically trapped and moved to roughly the center of the cell. The trapping beam was then blocked, and the sphere began to fall. When the sphere passed through the point where it came into focus when viewed through the microscope, it was followed while focus was maintained for a fixed time interval. The sphere terminal velocity was then obtained from the measured time interval and the travel distance of the microscope stage. Since the viscosity of water has significant temperature dependence, the gradual heating of the sample cell over the space of an hour from approximately $20{ }^{\circ} \mathrm{C}$ to $34^{\circ} \mathrm{C}$ by the laser beam and an incandescent lamp used to illuminate the sphere for microscope viewing was monitored by a type $\mathrm{E}$ thermocouple attached to the bottom of the slide. The measured value of the terminal velocity, ranging between 2.7 and $3.8 \mu \mathrm{m} / \mathrm{s}$, agreed well with the calculated terminal velocity assuming Stokes drag, including buoyancy, and by use of the published temperature dependence of the viscosity of water. ${ }^{33}$

We measured the trapping length of the laser beam by trapping a single sphere and moving it so that its surface was a predetermined distance $\Delta$ (between 4 and $50 \mu \mathrm{m}$ ) below the coverslip, blocking the beam for a fixed time interval during which the sphere fell through the sample cell, and then seeing whether the sphere was pulled upward to its original stable trapping position when the beam was unblocked. The distance of fall was obtained from the measured time interval and the sphere terminal velocity calculated assuming Stokes flow and by use of the published value of the viscosity of water for the temperature of the cell at the time experiment was performed. The experiment was repeated a number of times for each value of the starting distance and each time interval. Figure 7 shows the resulting inferred trapping length of the beam as a function of $\Delta$ based on a $100 \%$ retrapping rate. For $\Delta=4 \mu \mathrm{m}$, the inferred trapping distance is $23 \mu \mathrm{m}$, which is over a factor of 3 larger than the theoretical predictions of Section 3. However, for only slightly larger $\Delta$ the inferred trapping length rapidly decreased and leveled off at $\sim 7 \mu \mathrm{m}$, in nominal agreement with the theoretical predictions. A $100 \%$ retrapping rate was not achieved for $\Delta$ $>30 \mu \mathrm{m}$. We conjecture that the anomalously long inferred trapping lengths for $\Delta=4$ and $5 \mu \mathrm{m}$ result from either electrostatic interactions between the PSL sphere and glass coverslip or hydrodynamic interactions with the coverslip that delayed either the onset of the sphere's motion or its approach to terminal velocity. No additive was included in the PSLwater suspension to screen any electrostatic charge the spheres might have. For $\Delta=4 \mu \mathrm{m}$, hydrodynamic interactions with the coverslip ${ }^{34}$ increase the drag force by a factor of $\sim 1.6$ over Stokes drag, while for $\Delta=10 \mu \mathrm{m}$ the increase is only a factor of $\sim 1.1$. Additional evidence along these lines is provided by the fact that, when the sphere was in contact with the bottom of the coverslip and the trapping beam was blocked, it remained in contact with the coverslip for at least $20 \mathrm{~s}$ before starting to fall, and often had to be dislodged from the coverslip by a gentle tap on the slide.

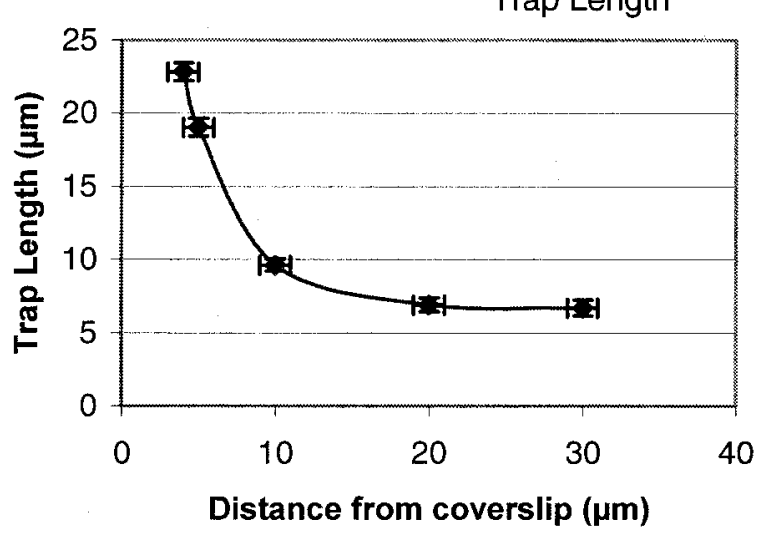

Fig. 7. Measured trapping length of the laser beam as a function of the distance of the PSL sphere's surface from the bottom of the glass coverslip, based on a $100 \%$ retrapping rate. 

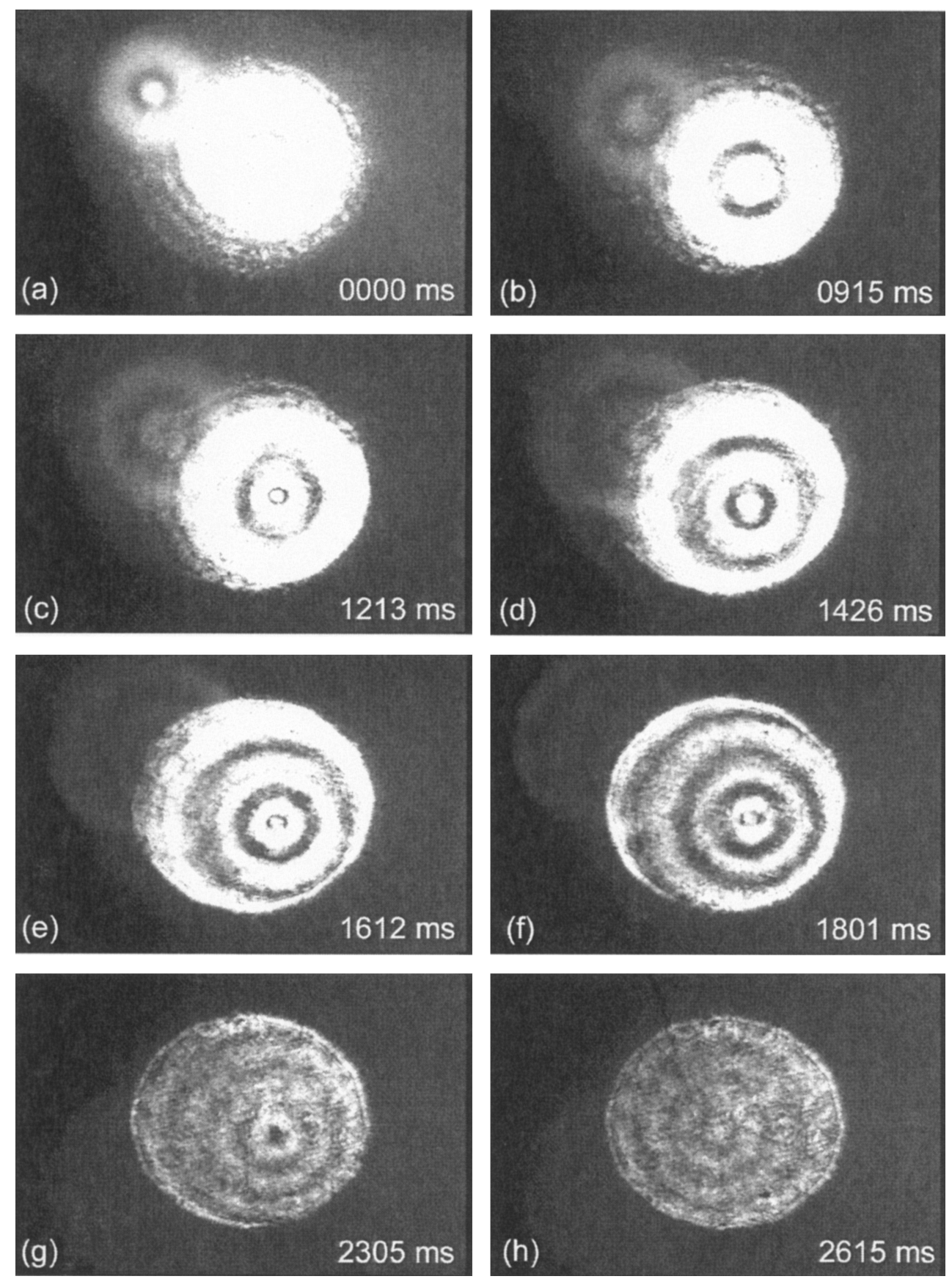

Fig. 8. Near-forward beamplus-scattered intensity at various times as the PSL sphere is pulled back up to its stable trapping position: (a) $0.000 \mathrm{~s}$, (b) $0.915 \mathrm{~s}$, (c) $1.213 \mathrm{~s}$, (d) $1.426 \mathrm{~s}$, (e) $1.612 \mathrm{~s}$, (f) $1.801 \mathrm{~s}$, (g) $2.305 \mathrm{~s}$, and $(\mathrm{h}) 2.615 \mathrm{~s}$.

During some of the retrapping experiments in which the PSL sphere had fallen to near the end of the trapping length before the beam was unblocked, the near-forward-scattered light passed through a beam splitter and illuminated a screen where it was recorded in video format as the sphere was pulled back up to its stable trapping position. Figure 8 shows a number of frames from a typical video sequence. In the earliest frame, Fig. 8(a), the sphere has fallen sufficiently far so that the beam focal waist lies a few micrometers outside it, and the nearforward light scattered by the diverging beam is very bright and relatively featureless. As the sphere is retrapped and moves upward toward its stable trap- ping position as in Figs. 8(b)-8(d), concentric interference fringes similar to those of Figs. 3(c) and 5(c) begin to form and give the appearance of propagating radially outward from the center of the pattern. As the sphere approaches the stable trapping position and the beam focal waist moves inside the sphere, the number of concentric interference rings continues to increase and the pattern generally becomes dimmer, as is observed in Figs. 8(e)-8(h) and predicted in Figs. 5(a) and 5(b) for the AFA beam model. By the time the sphere returned to its stable trapping position about six or seven pairs of bright and dark fringes had formed, in general agreement with Figs. 5(a) and 6. These video images show that, whereas the trapping 
properties of the Gaussian and AFA beam models are similar, only the latter model correctly predicts the angular structure of the near-forward scattered light. A similar scattering structure is apparent in Fig. 1(b) of Ref. 1 , in which the scattered light was viewed from the side, rather than head on.

\section{Conclusions}

Theoretically, although the trapping ranges and efficiencies of the highly idealized Gaussian beam and the more realistic AFA beam are quite similar, scattering by these beams exhibits large differences when the particle is held in the stable trapping position for high laser power. The Gaussian beam focuses deep inside the particle and the entire beam fits through the particle's effective aperture. As a result, the scattered light is also roughly Gaussian and the forward intensity remains slowly varying until the magnitude of $z_{0}$ increases enough so that the beam focuses outside the particle. The AFA beam field contains a long slowly decreasing tail in its focal plane that is due to the beam's sharp angular cutoff in the far zone. As a result, no matter whether the beam focuses inside or outside the particle, the particle's effective aperture truncates the beam, producing diffractive intensity ripples in the far zone. Our experimental results confirm these predictions and provide further evidence that Mie theory, augmented by a realistic model of the beam-shape coefficients, is capable of accurately predicting both the trapping and scattering properties of tightly focused, as well as paraxial, beams.

This research was sponsored by the Sub-sonic Fixed Wing Program at the NASA-Glenn Research Center under grant NAG3-2774.

\section{References}

1. A. Ashkin, J. M. Dziedzic, J. E. Bjorkholm, and S. Chu, "Observation of a single-beam gradient force optical trap for dielectric particles," Opt. Lett, 11, 288-290 (1986).

2. R. Gussgard, T. Lindmo, and I. Brevic, "Calculation of the trapping force in a strongly focused laser beam," J. Opt. Soc. Am. B 9, 1922-1930 (1992).

3. A. Ashkin, "Forces of a single-beam gradient laser trap on a dielectric sphere in the ray optics regime," Biophys. J. 61, 569-582 (1992).

4. Y. Harada and T. Asakura, "Radiation forces on a dielectric sphere in the Rayleigh scattering regime," Opt. Commun. 124, 529-541 (1996).

5. A. Rohrbach and E. H. K. Stelzer, "Optical trapping of dielectric particles in arbitrary fields," J. Opt. Soc. Am. A 18, 839 853 (2001).

6. A. Rohrbach and E. H. K. Stelzer, "Trapping forces, force constants, and potential depths for dielectric spheres in the presence of spherical aberrations," Appl. Opt. 41, 2494-2507 (2002).

7. In Ref. 13 it was incorrectly stated that Refs. 5 and 6 calculated the trapping force by use of Rayleigh scattering.

8. J. S. Kim and S. S. Lee, "Scattering of laser beams and the optical potential well for a homogeneous sphere," J. Opt. Soc. Am. 73, 303-312 (1983).

9. G. Gouesbet, B. Maheu, and G. Grehan, "Light scattering from a sphere arbitrarily located in a Gaussian beam, using a Bromwich formalism,” J. Opt. Soc. Am. A 5, 1427-1443 (1988).

10. J. P. Barton, D. R. Alexander, and S. A. Schaub, "Theoretical determination of the net radiation force and torque for a spherical particle illuminated by a focused laser beam," J. Appl. Phys. 66, 4594-4602 (1989).

11. K. F. Ren, G. Grehan, and G. Gouesbet, "Prediction of reverse radiation pressure by generalized Lorenz-Mie theory," Appl. Opt. 35, 2702-2710 (1996).

12. K. F. Ren, G. Grehan, and G. Gouesbet, "Radiation pressure forces exerted on a particle arbitrarily located in a Gaussian beam by using the generalized Lorenz-Mie theory, and associated resonance effects," Opt. Commun. 108, 343-354 (1994).

13. J. A. Lock, "Calculation of the radiation trapping force for laser tweezers by use of generalized Lorenz-Mie theory. 1. Localized model description of an on-axis tighty focused laser beam with spherical aberration," Appl. Opt. 43, 2532-2544 (2004).

14. J. A. Lock, "Calculation of the radiation trapping force for laser tweezers by use of generalized Lorenz-Mie theory. 2. On-axis trapping force," Appl. Opt. 43, 2545-2554 (2004).

15. B. Richards and E. Wolf, "Electromagnetic diffraction in optical systems. 2. Structure of the image field in an aplanatic system," Proc. R. Soc. London A 253, 358-379 (1959).

16. P. Torok, P. Varga, Z. Laczik, and G. R. Booker, "Electromagnetic diffraction of light focused through a planar interface between materials of mismatched refractive indices: an integral representation,” J. Opt. Soc. Am. A 12, 325-332 (1995).

17. P. Torok, P. Varga, Z. Laczik, and G. R. Booker, "Electromagnetic diffraction of light focused through a planar interface between materials of mismatched refractive indices: An integral representation: Errata,” J. Opt. Soc. Am. A 12, 1605 (1995).

18. J.-P. Chevaillier, J. Fabre, G. Grehan, and G. Gouesbet, "Comparison of diffraction theory and generalized LorenzMie theory for a sphere located on the axis of a laser beam," Appl. Opt. 29, 1293-1298 (1990).

19. F. Gilloteau, G. Grehan, and G. Gouesbet, "Optical levitation experiments to assess the validity of the generalized LorenzMie theory," Appl. Opt. 31, 2942-2951 (1992).

20. J. A. Lock and J. T. Hodges, "Far-field scattering of an axisymmetric laser beam of arbitrary profile by an on-axis spherical particle," Appl. Opt. 35, 4283-4290 (1996).

21. J. A. Lock and J. T. Hodges, "Far-field scattering of a nonGaussian off-axis axisymmetric laser beam by a spherical particle," Appl. Opt. 35, 6605-6616 (1996).

22. G. Arfken, Mathematical Methods for Physicists, 3rd ed. (Academic, 1985), p. 604, Eqs. (11.85) and (11.86).

23. H. C. van de Hulst, Light Scattering by Small Particles (Dover, 1981), p. 123.

24. H. M. Nussenzveig, "High-frequency scattering by a transparent sphere. 1. Direct reflection and transmission," J. Math. Phys. 10, 82-124 (1969).

25. E. A. Hovenac and J. A. Lock, "Assessing the contributions of surface waves and complex rays to the far-field Mie scattering by use of the Debye series," J. Opt. Soc. Am. A 9, 781-795 (1992).

26. L. Brillouin, "The scattering cross section of spheres for electromagnetic waves," J. Appl. Phys. 20, 1110-1125 (1949).

27. B. Maheu, G. Grehan, and G. Gouesbet, "Ray localization in Gaussian beams," Opt. Commun. 70, 259-262 (1989).

28. J. A. Lock, "Contribution of high-order rainbows to the scattering of a Gaussian laser beam by a spherical particle," J. Opt. Soc. Am. A 10, 693-706 (1993).

29. M. Abramowitz and I. A. Stegun (eds.), Handbook of Mathematical Functions (National Bureau of Standards, 1964), p. 487, Eq. (11.4.42).

30. H. M. Nussenzveig and W. J. Wiscombe, "Forward optical glory," Opt. Lett. 5, 455-457 (1980).

31. D. S. Langley and M. J. Morrell, "Rainbow-enhanced forward and backward glory scattering," Appl. Opt. 30, 3459-3467 (1991). 
32. J. A. Lock and T. A. McCollum, "Further thoughts on Newton's zero-order rainbow," Am. J. Phys. 62, 1082-1089 (1994).

33. R. C. Weast (ed.), Handbook of Chemistry and Physics, 50th ed. (CRC, 1969), p. F36.
34. J. Happel and H. Bremmer, Low Reynolds Number Hydrodynamics with Special Applications to Particulate Media (Prentice-Hall, 1965), p. 330, Eqs. (7.4.37)-(7.4.39) and p. 331, Table 7.4.1. 